\title{
Properties and Dispositions: Some Metaphysical Remarks on Quantum Ontology
}

\author{
Mauro Dorato \\ Department of Philosophy, University of Rome3, Via Ostiense 234 \\ 00199, Rome, Italy \\ e-mail:dorato@uniroma3.it
}

\begin{abstract}
After some suggestions about how to clarify the confused metaphysical distinctions between dispositional and non-dispositional or categorical properties, I review some of the main interpretations of QM in order to show that - with the relevant exception of Bohm's minimalist interpretation - quantum ontology is irreducibly dispositional. Such an irreducible character of dispositions must be explained differently in different interpretations, but the reducibility of the contextual properties in the case of Bohmian mechanics is guaranteed by the fact that the positions of particles play the role of the categorical basis, a role that in other interpretations cannot be filled by anything else. In Bohr's and Everett-type interpretations, dispositionalism is instrumentalism in disguise.
\end{abstract}

Keywords: Dispositional Property, Categorical Property, GRW, Many-Worlds, Relationism, Bohr, Rovelli, Bohm

PACS: 03.65.-W

\section{INTRODUCTION: WHY SHOULD WE TALK ABOUT PROPERTIES IN QM?}

As a philosopher of science, I think that the best and most honest way to celebrate Gian Carlo Ghirardi $70^{\text {th }}$ birthday is to offer some metaphysical remarks on a remarkable aspect of the quantum world, namely the radical relativization of the notion of physical property that quantum mechanics (henceforth, QM) has forced upon us, independently of the various interpretations. It may seem even obsolete to try to talk about "properties" in the holistic, contextual setting of QM, where "contextual" has of course a precise meaning, derived from well-known no-go theorems against the possibility of simultaneously assigning non-contextual and definite values to a physical system whose Hilbert space has dimensions greater than two [1].

Let me clarify at the outset that the notion of "property" I advocate is not so speculative or metaphysical: the idea is that a physical system possesses a property when some observable $A$ has a definite, precise magnitude within the margins of experimental error. Think of the mass or the charge of an electron, or of the intensity 
of the electric field in a given space-time region, as examples of "kosher" physical properties, where kosher means that they are non-contextually and intrinsically possessed by the electron and the field respectively. Such properties are intrinsically possessed by electron and the field in the sense that their magnitude is independent of the test particles that we must necessarily use to measure them. It seems to follow that if talking about a physical property presupposes a certain degree of intrinsic-ness or independence of the property from whatever else exist in the world, then we may well have to conclude that the notion of property, as it was normally presupposed in classical physics, in QM undergoes, to say the least, a radical change which deserves some further investigation. If (i) contextuality is ubiquitous across interpretations as different as Bohm's, Everett's, GRW's and Bohr's, and if (ii) the contextuality of a property $x$ is equivalent to the fact that the system lacks $x$ altogether, why still talk of microentities possessing dispositional properties? Isn't the very talk of property the residue of an old metaphysics that we should dispose of?

In order to inquire into the alleged dispositional character of the quantum world and try to answer the question above, I begin by clarifying some problematic metaphysical distinctions between intrinsic and relational properties on the one hand, and occurrent or categorical and dispositional properties on the other. Then I review some of the main interpretations of QM (Bohm, GRW, Everett/Rovelli and Bohr), in order to show to which extent they are committed to dispositional properties. It will turn out that the only theory in which dispositions and relations are reducible to categorical properties is Bohm's, and this is especially evident in the so-called "minimalist" interpretation due to the "trio" [3]. On the one hand, the fact that dispositions play a (albeit different) role in interpretations as different as Bohm, Bohr's, Everett's and GRW's might tempt one to claim that dispositionality is a feature of the micro-world that is here to stay. On the other, the presence of irreducible dispositions might be regarded as the safest piece of evidence of the fact that we still don't understand QM.

\section{DISPOSITIONAL, RELATIONAL AND CONTEXTUAL PROPERTIES}

Just to motivate an incursion into the metaphysics of properties, consider that talking of "properties" cannot easily be dispensed in the context of QM, since, at least prima facie, in unified dynamical models like those proposed by Gian Carlo Ghirardi and collaborators, it plays a pivotal role [2]. After all, the measurement problem consist in finding out in which circumstances we are allowed to attribute a definite property to a physical system.

Let us begin by considering that physics is full of dispositional properties (in short dispositions): think just of the paradigmatic cases of "fragility" or "permeability". These dispositions are characterized by the fact that they become manifest and observable only in certain contexts. "Being broken", or "being wet" are not only the manifestations of these dispositional properties, but are also instances of categorical or non-dispositional properties. Intuitively, a disposition like permeability is not 
directly observable all the times, as is the property given by the form of an object ("being spherical"), but becomes observable only when the entity possessing it interacts with water or other fluids. Why should we worry about dispositions in QM?

Typically, a dispositional property of glass, like "fragility", which is a macroscopic property, is reducible to, identical with, or explainable in terms of, the atomic/molecular structure of the glass, referred to as the microscopic, categorical basis of the disposition, plus the breaking context (the stone hitting the glass). Likewise, being flexible or being a good conductor, qua dispositional, macroscopic properties of copper, are all regarded as being reducible to, identical with, or explainable in terms of, the atomic structure and forces holding together the microscopic components of copper. The question I want to raise in this paper is whether and in what sense QM, in its various interpretations, forces us to accept the existence of ungrounded, irreducible, probabilistic dispositions, i.e. dispositions, that, unlike fragility or permeability, lack any categorical basis to which they can be reduced to. My claim is that the presence of irreducible quantum dispositions in many (but not all) interpretations involves the difficulty of giving a spatiotemporal descriptions to quantum phenomena, and is therefore linked to our lack of understanding of the theory, i.e., of our lack of a clear ontology underpinning the formalism. Before reviewing the various interpretations from this perspective, however, it is important to clarify the ground from possible confusions between relational, dispositional, contextual and their antonyms, namely, intrinsic, categorical and non-contextual properties.

Consider dispositions like "irritable" or "poisonous", which manifest themselves in appropriate contexts, when people get angry and mushrooms poison the blood. From these ordinary language examples, it would seem that the function of dispositional terms in natural languages is to encode useful information about the way objects around us would behave were they subject to causal interactions with other entities (often ourselves). This remark shows that the function of dispositional predicates in ordinary language is essentially predictive. Consider the evolutionary advantage of classing all animals or people around our ancestors as "dangerous" or "innocuous", as "peaceful" or "ferocious". In learning that a particular mushroom is "poisonous", a child learning the language also learns to stay away from it whenever she recognizes one.

I think that the predictive role of dispositional terms is the main explanation of the reason why natural languages are so replete with dispositional terms, which refer to microscopic, inner non-directly observable properties, and also point, more or less directly, to the complex relationships linking dispositions with causes, counterfactuals and, eventually, laws of nature as they are expressed in our scientific languages. A stone causes the manifestation of the disposition "fragile" (and therefore causes the breaking of the glass) because it causally interacts with its microscopic structure. Counterfactuality is involved because attribution of the disposition fragility to the glass entails that were it struck in an appropriate way, the glass would break, while the regularity with which the fragility of glasses is manifested refers to a law of nature capturing the behavior of the micro-constituents of glass. In a word, dispositions

${ }^{1}$ The choice between these three options is highly debated in the philosophical literature on dispositions, but here we can avoid to take a stand among them. 
express, directly or indirectly, those regularities of the world around us that enable us to predict the future. Such a predictive function of dispositions should be attentively kept in mind when we will discuss the "dispositional nature" of microsystems before measurement, in particular when their states is not an eigenstate of the relevant observable. In a word, the use of the language of "dispositions" does not by itself point to a clear ontology underlying the observable phenomena, but, especially when the disposition is irreducible, refers to the predictive regularity that phenomena manifest. Consequently, attributing physical systems irreducible dispositions, even if one were realist about them, may just result in more or less covert instrumentalism.

The "epistemic cash-value" of dispositional terms also points to another, related feature of dispositions, first stressed by the founders of modern mechanical philosophy, but already known to the ancient atomists. This feature corresponds to the fact that dispositional terms, besides expressing predictions, also refer to the way the world "in itself" appears or manifests itself to our senses. "Odorous" and "stinking", referred to a rose and to rotten meat, "sweet" and "sour", referred to sugar and lemons, "red" and "white", referred to two flowers, for philosophers like Galileo, Boyle, Descartes and Locke, do not point to properties existing in themselves (independently of our minds), but to the products of the interaction between the "primary", intrinsic qualities of the objects around us (including their intrinsic dispositions) and our sensory system (they way such dispositions manifest themselves to sensory beings like ourselves).

This second role that dispositions have had in the philosophical language seems to call attention to the fact that human beings filter and respond selectively to the mindindependent properties of the external world, either through their nervous system or their minds. And this sense has also been regarded as important in all those interpretations in which human consciousness is explicitly invoked to solve the measurement problem $[4,5] .^{2}$ The difference with the primary/secondary quality distinction proposed by modern philosophers is that dispositions evoking in our minds secondary properties or "qualia" such as red, hot, sweet, could still be referred to primary qualities like the shape, the number and other categorical properties of corpuscles or atoms, which were their categorical basis. When a system is in a superposition of states, however, it is not at all clear which definite, categorical properties could form the basis for our perception of pointers being in a definite position.

Notice that in both of their functions, the ordinary and the more philosophical one, the manifestation of dispositional properties presupposes causal interactions of some sort: the property of "being soluble" needs an interaction with some liquid to manifest itself, in the same sense in which the fragrance of a flower needs the interaction of certain chemicals with human nostrils and rhinencephalons for its manifestation. Despite the relational nature of the manifestation of dispositions, it would be a mistake to identify the non-dispositional or categorical properties with the merely intrinsic properties of an object, namely with those properties whose exemplification by an entity does not presuppose the existence of any other entity. ${ }^{3}$ In order to avoid

\footnotetext{
${ }^{2}$ As a matter of fact, later in life Wigner tried to propose non linear modifications of Schrödinger’s equation. [6. p.339].

${ }^{3}$ To understand the difference, compare being square, which is intrinsic, with being simultaneous with, which is relational. The first predicate is monadic, as it needs only one filler, while the second is at least two-placed.
} 
misleading confusions, it is important to stress that "dispositional" and "relational" are to be regarded as logically independent notions, exactly like their opposites, "categorical" and "intrinsic" [7, p. 69]. Granted that we must distinguish the possession of a property from our way of ascertaining that such a property is possessed - something which always calls into question a causal or an experimental context - charge, spin and mass should be regarded as intrinsic, non-relational properties of microscopic particles. One could be tempted to claim that being intrinsic is at least sufficient for being non-dispositional, so that "being dispositional" might entail "being relational" (the contrapositive claim). However, it makes perfect sense to claim that my window pane is fragile even if it never breaks and that it would continue to remain fragile even if no other object were around. If we distinguish, as we should, the possession of a property from our way of ascertaining such a possession, a glass would be fragile even if it were the only object in the universe, so that it could never break for "lack of stones".

In this sense, any disposition can be regarded as an intrinsically possessed property, so that being intrinsic is not sufficient for being categorical. Equivalently, if a disposition can be, and usually is, intrinsic, it follows that relationality is not necessary to dispositionality. And neither is it sufficient, as the trivial example of "being the son of", a relation that is not a disposition, clearly shows. Against Popper, therefore, who treated dispositions or probabilistic propensities as objective but relational features of the whole experimental set-up [8], we should distinguish the possession of a dispositional property, which has a certain probability of manifesting itself in a determinate way, from such a manifestation, which is clearly an event, a causal process, (the breaking of the glass, the hitting of a quantum particle on a fluorescent screen, etc) and not a property. The categorical property "broken" or "located on a spot" is somehow the conclusion of the process of manifestation of the disposition.

Finally, we should ask what is the conceptual relationship between the attribution of dispositions and contextuality as it emerges in Kochen-Specker's hypothesis and similar no-go results. The idea of contextuality is simple: if we assign a certain value to the square of the operator "spin in the $z$ direction" - call it $\mathrm{S}^{2}{ }_{\mathrm{z}}$ - when it is measured together with $\mathrm{S}_{\mathrm{x}}^{2}$ and $\mathrm{S}_{\mathrm{y}}^{2}$ in the direction $x$ and $y$, if $\mathrm{S}_{\mathrm{z}}^{2}$ is not contextual we must get the same value if we measure it together with $S^{2}{ }_{x}$, and $S^{2}$, assuming that the direction $x^{\prime}$ and $\mathrm{y}^{\prime}$ are different from $x$ and $y$. Therefore, contextualism seems to entail that some QM “properties” are not possessed before measurement, since otherwise they could not manifest themselves in different ways according to the type of measurement we perform. ${ }^{4}$

This fact seems to establish a strong link between contextuality and dispositionality. Within QM, it seems natural to replace "dispositional properties” with "intrinsically indefinite properties", i.e. with properties that before measurement are objectively and actually "indefinite" (that is, without a precise, possessed value). So the passage from dispositional to non-dispositional is the passage from the indefiniteness to the definiteness of the relevant properties, due to measurements interactions.

\footnotetext{
${ }^{4}$ In different words, we cannot assume that there is a one-to one correspondence between an operator and an observable, so that contextualism bans a certain form of naïve realism about operators [9].
} 
Of course, in those situations in which the system possesses a precise value of a certain observable (property) even before measurement (when its state is an eigenstate of the observable), the measurement interaction provided by the experimental context simply amplifies the microscopic value to a macroscopic, classical scale.

Consequently, in QM we seem to have two kinds of intrinsically possessed properties, depending on the way the system has been prepared before measurement: if the system has a definite value also before measurement and the latter just reveals it, the system possesses a categorical property, which the apparatus simply reveals. On the contrary, if the value revealed by the measurement interaction causally depends on, or is even created by, the interaction, we have a form of contextualism which implies the presence of intrinsic dispositions [10, p. 283]. In this second case, the idea is that the property that is experimentally manifested by the microsystem depends on the measurement context.

Consequently, following in part [10] - who distinguish between two forms of contextuality, a weak and a strong form - I propose to redefine the classical distinction between dispositional and non-dispositional (categorically possessed) properties simply in terms of the difference between states of quantum systems that are and states that are not in an eigenstate of the relevant observable.

Before examining the main interpretations of QM with the purpose of understanding the role played by reducible and irreducible dispositions, I must face a couple of foreseeable objections that were briefly voiced in the introduction. Why should we attribute intrinsic dispositions to have a spin in the $z$ direction to, say, particles in a superposition of spin up in the $x$ direction and spin down in the $x$ direction, given a certain orientation of a Stern-Gerlach magnet? Isn't it more appropriate to claim that a micro-system in such a superposed state of spin before measurement simply has no spin at all in the $z$ direction? The first objection, in a word, is that, at least in the context of QM, "properties that are merely contextual (i.e., dispositional in our language) are no properties at all, they do not exist, and their inadequacy to play the role of properties is meant in the strongest possible sense" [6, p. 330, my transl.]. In addition, what do we gain, for purposes of explanation and prediction within, say, GRW type of theories, by attributing isolated microsystems an intrinsically, non reducible disposition to localize, over and above writing down the law that explains how and when localizations occur?

In the case of contextual/dispositional properties like spin direction in the Bohmian interpretation, on which we will return in the following sections, I will just say this: affirming that a microsystem has a disposition to manifest a certain spin along the $\mathrm{z}$ direction is consistent with, and implied by, the claim that before measurement it has no categorical property of that type. The attribution of the disposition to the microsystem in question at this stage has just the purpose of reminding us that what we observe in a quantum experiment is often the product of an interaction between some property, tendency or capacity of the microsystem (however we want to call the possession of magnet-like structure) and the apparatus, in such a way that what we observe after the interaction was not there before. Analogously, mechanisms of spontaneous localizations of the wave functions in certain GRW models are consistent with claiming that before localizing, microsystems have just a tendency to localize but 
possess no definite position at all. Such a difference with classical physics is worth noting at least on a first analysis, and even Bohm and Hiley have written that "The context dependence of results of measurements is a further indication of how our interpretation does not imply a simple return to the basic principles of classical physics.”[11, p. 9]. The advantage of using the word "dispositions” in such cases lies simply in creating the presupposition for a conceptual confrontation of QM with classical physics, and nothing more "metaphysical" than that. With this clarification, I think that the use of a dispositional talk will not be misleading.

As to the second criticism, we learned from the debate on disposition that we cannot analyze fragility in a conditional way ("fragile" does not mean that "if appropriately struck it breaks"), since a piece of glass remains fragile even if it will never break. Therefore, a reducible disposition is something more than a predictive correlation expressed by a material conditional "if...then..."; but what can we say about the irreducible disposition of a single proton to localize? Is there something to it more than the phenomenological law describing the average process?

In order to test the validity of the first reply and try to answer the question above, it will best to examine the different role that dispositions play in QM, the main difference being not their presence in the various interpretation, but their reducibility. In having chosen to present only some of the most discussed interpretations of QM, I readily admit that my review does not exhaust all possible ways of understanding it ${ }^{5}$ : my aim is rather to try to understand how fruitful and unifying the perspective offered by dispositions can be in making sense of the ontology of the theory.

\section{“DISPOSITIONALIST” READINGS OF QM}

Interestingly, we find dispositionalist views of QM both in subjectivist and in objectivist interpretations. To begin with the latter, Karl Popper has often regarded his propensity interpretation of QM as his major contribution to the philosophy of physics, and of QM in particular [8]. Both he and Nicholas Maxwell after him [14] have defended some sort of a propensity interpretation of probability, and a view of quantum reality essentially characterized by irreducibly probabilistic but real propensity (propensity waves or propensitons). On the former camp, instead, Werner Heisenberg had made direct reference to Aristotelian potentiae to refer to and interpret the elusive nature of the atomic and subatomic world before measurement: "Such a probability function [i.e. the statistical algorithm of quantum theory] combines objective and subjective elements. It contains statements on possibilities, or better tendencies ("potentiae" in Aristotelian philosophy), and such statements are completely objective, they don't depend on any observer...the passage from the "possible" to the real takes place during the act of observation» [12, pp. 67-69]. It should be noted in passing that here Heisenberg might have described also the measurement interaction in a less subjective way, by insisting that "the passage from the possible to the real" occurs in any interaction with a measuring instrument, and not

\footnotetext{
${ }^{5}$ For instance, I have omitted the modal interpretations, in which propensities may have a certain role [16, 17].
} 
just with human observers. After all, unless one expects from consciousness a special but illegitimate help to solve the measurement problem, within the context of that problem "observers" should be regarded simply as highly complex physical systems: one cannot solve a mystery by invoking another mystery. When Heiseberg claims that potentialities are objective, he means that the probabilities that measure them are not subjective, but express objective chance.

Some years before, Henry Margenau had already characterized the properties of quantum systems as merely latent, in contrast to the properties intervening in the description of classical systems, which are always definite even when they are merely dispositional [13, p. 6]. In discussing Bohm's contextualism, we will see that this statement is essentially flawed.

Probably influenced by this tradition, Michael Redhead's influential textbook on the philosophy of QM distinguishes among three different interpretations of the theory, the second of which presupposes real propensities and potentialities and attributes to measurements the function of «converting latent values in possessed values» $\left[15\right.$, p. 48]. ${ }^{6}$ Clearly, for this second interpretation to make more than an instrumentalistic sense, one needs to construe potentialities and dispositions as real properties of systems. More recently, Mauricio Suárez has defended a new propensity interpretation of QM [16, 17] against two objections by Grossman [18] and Milne [19]. In his view, a quantum propensity is intrinsic to the quantum system (it is independent of the experimental set-up that is designed to make it manifest), and it is only the manifestation of the property that is relational. Furthermore, he attaches an important meaning to the dispositionality view by linking it to the measurement problem.

Despite this literature (see also [10]), what seems to be lacking, however, is a more systematic survey of the territory covered by dispositions within the main interpretations of QM; this seems important in order to get a firmer grasp of the dispensability or the centrality of a dispositional talk in understanding the nature of those states that are not eigenstates of the relevant observable.

\section{THE REDUCIBILITY OF DISPOSITIONS IN BOHM'S MINIMALIST INTERPRETATION}

As is well known, in David Bohm's mechanics, QM is regarded as incomplete, and is supplemented by an additional but "not so hidden" variables, the changing-in-time positions of all particles composing a physical system. Such a variable is obviously epistemically inaccessible before a measurement, but since it is postulated to be definite at all times, it can be revealed by faithful measurements.

The theory consists by two equations, the familiar Schrödinger's deterministic differential equation:

\footnotetext{
${ }^{6}$ The first position he discusses is the Copenhagen interpretation, while the third is associated with a "property realism" of Bohm's type. For these interpretations, see below.
} 


$$
i \hbar \frac{\partial \Psi}{\partial t}=\hat{H} \Psi
$$

and an additional "guidance equation", relating non-locally the velocity of the k-th particle to the positions $\mathrm{Q}=\left(\mathrm{Q}_{1}, \ldots . . \mathrm{Q}_{\mathrm{n}}\right)$ of all the others, and more precisely, to the gradient of the $\psi$-function with respect to the k-coordinate of the particle:

$$
\frac{d Q_{k}}{d t}=v_{k}^{\Psi}=\frac{\hbar}{m_{k}} \operatorname{Im}\left(\frac{\nabla \Psi_{k}}{\Psi}\right)\left(Q_{1}, Q_{2} \ldots Q_{n}\right)
$$

Besides its deterministic features, for our purpose it is of paramount importance to remind the reader that (i) this interpretation divides the physical observables into two categories, the non-contextual or categorically possesses properties, and the contextual or dispositional properties, and that (ii) position is regarded as the only categorical property. As we know, in our terminology this essentially means that position is the only variable that can be regarded as being possessed before measurement and independently of measurement: the choice of position as the only categorical property is suggested by the practical need to explain our definite macroscopical observations (the choice of momentum as a categorical property would be an open theoretical possibility but lack in explanatory power). It is easier to understand why a pointer is there rather than in a macroscopic superposition of being "here and there" if its particles are always in a definite position.

Furthermore, the categorical aspect of position has another extremely important aspect, linked to the centrality that spacetime assumes in Bohmian mechanics. Clearly, the possession of a definite position by all particles at all times implies that QM is describable within a definite spatiotemporal arena (Newtonian spacetime, regarded as a collection of worldlines). Of course, I am not claiming that all categorical properties are spatiotemporal or geometrical properties, even though the form or the shape of an object are paradigmatic examples of non-dispositional properties. Rather, I am arguing that the non-dispositional character of position "forces" Bohmian mechanics to give spacetime a preeminent role. This might have far reaching consequences in view of a quantum theory of gravity: it seems difficult that spacetime can emerge from a quantum domain, if the latter requires the former to make sense of its ontological posits. Of course, abandoning localized particles as approximation in another regime (say, the relativistic regime) would substantially weaken my claim, but we don't have so far a fully developed relativistic Bohmian mechanics.

The dispositional/contextual features of this interpretation follows from the fact that the wave function depends in a non-local way on the positions of the particles composing the system, as well as on the whole experimental setup. ${ }^{7}$ In order to be more specific, suppose that we are trying to measure along the $\mathrm{z}$ direction the spin of a particle that is in a superposition of being up and down in the $z$ direction with equal

\footnotetext{
${ }^{7}$ Such a contextualism is a welcome consequence of Bohm's theory, since the contrary assumption that all properties of a quantum system have definite, possessed values that are independent of the measurement context would lead to a contradiction with the above mentioned Kochen and Specker's theorem [1].
} 
probability. If we invert the polarity of the Stern-Gerlach apparatus by leaving everything else unchanged, we change the measurement result of spin in the $z$ direction: if we had obtained $z$-up in the previous measurement, now we obtain $z$ down and vice versa. To put it in a slogan, "positions being equal, different measurements yield different results".

Given this fact, the property of "having a definite spin in the $z$-direction" is not categorical or intrinsic, or faithfully measured: measurements do not in general reveal the pre-existing possessed value of spin along the $z$-direction, for the simple reason that there isn't any even in Bohm's interpretation! If by switching the magnet and leaving everything else unchanged we change the experimental outcome from $z$-up to $z$-down, prior to measurement the system has no definite property of having a spin in the $z$-direction. Before measurement, the property "spin along the $z$-direction" is therefore dispositional, for the simple reason that the experimental outcome causally depends on the measurement context together, of course, with the "hidden", categorical and non-contextual value of the position of the particle.

Exactly for this reason, however, in the Bohmian interpretation the dispositional properties of "spins along a given direction" - referred to particles that before measurement are in a state of superposition with respect to that direction - are reducible to position and the context of measurement [10, pp. 285-6]. The idea of reducibility is based upon the fact that our hypothetical knowledge of the position of the particle plus knowledge of the orientation of the magnet would enable us to deduce the experimental outcome with certainty.

We can now go back to Margenau's comment reported above. We have seen that also in Bohm's theory, which is the most realistic about possessed properties, there cannot be any pre-existing, real property of "having a spin in a certain direction" to be revealed by measurements along that direction. If in the quantum case measurement somehow creates the property, isn't this different from fragility, which is possessed in a definite degree by a glass also before the breaking context? In order to defend a perfect analogy between a Bohmian disposition "to have spin in a given direction" and classical dispositions like those possessed by a glass, however, one must simply point out that the latter's "propensity to break in a certain context" corresponds to "the propensity to manifest a definite spin in a certain measurement context". Anyone committed to the reality of dispositions would clearly maintain that such a propensity is possessed by Bohmian particles also before measurement, and independently of it. Consequently, in the Bohmian case at least, the correct analogy with the classical disposition "fragility" is not given by "having a definite spin" but by "the disposition to have a definite spin in a given direction", which is linked to the fact that elementary particles can be deflected by magnetic fields, analogously to tiny magnetic needles that in some circumstances do not point along a $z$ direction (when they are in a superposition of spin along that directions).

It follows that the manifestation of the two dispositions is, respectively, the breaking event in one case and the acquisition of a definite spin in the other. Accordingly, and even from the viewpoint of a realist position about dispositions, Clifton and Pagonis are correct in holding that the dispositionality of properties in Bohm's theory is "nothing to write home about", as it is not remarkably different from the dispositionality of ordinary classical properties. [10] 


\section{DISPOSITIONS IN GRW}

In the so-called dynamical reduction models of non-relativistic QM, to which we will refer - somewhat schematically and with apologies to the others contributors to this interesting research program - with $G R W$ (from the acronym of their main inventors, Gian Carlo Ghirardi, Alberto Rimini and Tullio Weber), the definite, macroscopic world of our experience, threatened to be in a nebulous state by the universal validity of the principle of superposition conjoined with the assumption of completeness of QM, is obtained via a modification of the linearity of Schrödinger's equation. ${ }^{8}$ In GRW's original model [21], on which we will focus here, the wave function of a system is multiplied by a localization function, which physically represents a spontaneous localization in a "limited" region of space of a previously non-localized quantum system.

According to this reduction model, the fundamentally stochastic nature of the localization mechanism is not grounded in any categorical property of the quantum system: the theory at present stage is purely "phenomenological", in the sense that no "deeper mechanism" is provided to account for the causes of the localization. "Spontaneous", as referred to the localization process, therefore simply means "uncaused". This remark is going to be of fundamental importance to provide evidence in favor of the view that in this interpretation of QM, localization mechanisms are ungrounded or irreducible, probabilistic dispositions. However, despite their irreducibility, such dispositions can be measured and have a predictive import, as they can tell us in a precise way how often and at what scale does a localization occur.

In the attempt to unify the dynamics of microscopic and macroscopic systems, in [21] GRW suppose in fact that all individual quantum systems have an irreducibly probabilistic disposition to localize in a region of space whose dimension is approximately $10^{-5} \mathrm{~cm}$, with a frequency $f$ given by $10^{-16} \mathrm{sec}^{-1}$. The probability that such a process occurs is approximately once every hundred million years, and this hypothesis explains why isolated quantum systems can typically remain for a very long time in non-localized or superposed state (i.e., they are "spread" across a large region of space).

However, since a macroscopic system is constituted in average by $10^{23}$ atomic components, and since in GRW's model the localization of a single particle drives the collapse of all the others, it follows that the components of a macroscopic apparatus (a pointer) that are correlated with the particle that we want to measure will undergo a localization every $10^{-7}$ seconds. In fact, the average number of particles that will collapse spontaneously in a second is given by $10^{-16} \times 10^{23}=10^{7}$, which means that the macroscopic apparatus remains in a state of indefinite position (i.e., in a superposition

\footnotetext{
${ }^{8}$ A recent, very accurate survey of dynamical reduction models is given in [20].
} 
of two position states) for no more that $10^{-7}$ seconds: as Bell put it, Schrödinger's cat remains neither dead nor alive for no more than a split second [22, p. 44].

For our purposes, it is essential to stress once again that the new uncaused tendency to "swerve" attributed to atoms by GRW (in Lucretius' De Rerum Natura we read of a "clinamen" accidentally deviating the vertical fall of the Democritean atoms $)^{9}$ is an irreducibly dispositional property, that becomes actual or is manifested in ways that could call for - were the new theory prove to be successful in overcoming its present difficulties with a relativistic extension - the introduction of new constants of nature.

Furthermore, despite the fact that the irreducibly stochastic propensity to localize is not grounded in any categorical properties of the quantum system, it is nevertheless strongly explanatory of the definiteness of the macroscopic world of our experience. In this case, and using the familiar example from scholastic philosophy, we are not facing virtutes dormitivae which tautologically "explain" why opium makes you sleep. Consequently, and this is important especially for the philosophical debate, we should change our prejudices concerning ungrounded dispositional properties as being always explanatorily empty. The remarkable explanatory power of GRW type models is given by the unification that the modified Schrödinger's equation achieves between the dynamical evolution of quantum systems and the classical evolution of macroscopic systems, in accord with well-known philosophical theories which regard scientific explanations as unifications of phenomena under a single, more general law of nature [23, 24].

An ungrounded disposition to localize is therefore the fundamental ontological tenet of this interpretation of QM. Isolated particles of course exist, also before localizing, in the sense they are somewhere in spacetime, but the fact that they can be "here" as well as on the moon poses some methodological difficulty in the relationship of this version of GRW with an ontology of events. I am not thinking of the problem of giving a relativistic invariant version of the localizing process; I am thinking of the possibility of giving a spatiotemporal description of isolated entities prior to their localizations, in such a way as to have a well-funded notion of event, the building block of relativity. The difficulty seems to be that before a localization, there are no categorical spatial properties to which we can refer for assigning spatial relations. It is only via macroscopic objects that we can do so: chairs, rulers, table, trees just are the product of an amazingly large numbers of localizations events.

In a word, I am claiming that we cannot have an ontology of events before the localizations - even though we may have bare Newtonian spacetime (for substantivalists) - because we have no precise localizations for isolated particles. It is for this reason that, within an ontological option in which there are irreducible dispositions of the GRW type, it is reasonable to follow Bell [22] and assume that physical spacetime, regarded as the set of localized physical occurrences, is just constituted by or identical with the set of all "flashes", or localizations, occurring at a precise location at a certain time. These are events because they are well localized, and are endowed with sufficiently categorical properties (first and foremost a precise location in spacetime) for being the basis for a reconstruction of the properties of

${ }^{9}$ This historical parallel is due to Van Fraassen. 
tables and chairs. But it is important to notice that the notion of event allowed by the this version of GRW is macroscopical, not microscopical.

My claim is therefore that the irreducible character of localizations in GRW (in one of its interpretations) is equivalent to, or at least entails, the impossibility of giving a spatiotemporal description which can be used to explain or ground the localizations events. Rather, it is the collection of such localization events that constitute both physical spacetime and the world around us: as Bell put it, a piece of matter is a galaxies of such [localization] events [22, p. 204]. In a word, at least in this reading of GRW's ontology, spacetime is simply derived out of quantum events, a feature that might be interesting in quantum gravity programs, insofar as the latter requires that spacetime be a non-fundamental, derived, emergent notion.

We should recall that in another reduction model proposed by Ghirardi, the fundamental entity is a scalar field $\rho=\rho(\boldsymbol{r}, t)$ defined on Newtonian spacetime, with $\rho$ being, at macroscopic scale, what we call mass density of physical objects. In this interpretation, the wave function $\Psi\left(r_{1}, . . r_{n}, t\right)$ describes the system at a given time, and the square modulus of $\Psi$ of determines, for each particles $i$, how much stuff $\left(\rho_{i}\right)$ there is in a given cell:

$$
\rho(\mathbf{r}, t)=\sum_{i} m_{i} \rho_{i}(\mathbf{r}, t)
$$

In this view, some categorical properties are ascribed to the scalar field $\rho$ and even though the density of microscopic objects can be in a superposed state and enjoy "the cloudiness of waves”[17, p.349], due to the localization mechanisms the mass density of macroscopic objects (Schrödinger's poor cat) acquires a precise value in a split second, and the object localizes somewhere via an irreducibly stochastic event.

We conclude by pointing out that, from our chosen perspective, the main difference between Bohm's and GRW's interpretations lies in the different way in which they treat dispositional properties: reducible in the former case, irreducible in the latter. As a consequence, while in the former case Bohm's theory is about a family of trajectories in spacetime and spacetime is a fundamental notion - spacetime just is a class of histories of events with genidentical relations bearing with one another a relation of temporal succession - in the interpretation of GRW à la Bell, physical spacetime is a derived notion. In the "mass density reading" of reduction models, bare, "empty" spacetime is so to speak already presupposed for the scalar field to be defined on it, but what is acquired via the localization mechanism is the possibility of individuating macroscopic bodies via their location. Although the scalar field $\rho$ is in spacetime, also in this case the precise individuation that makes an ontology of events possible is realized only "after" the stochastic processes.

In a word, the irreducible, non-categorical nature of the fundamental properties postulated by GRW is linked to the fact that, in reduction models, physical spacetime is derived via the localization processes which are macroscopic events, while in Bohmian mechanics events can be as microscopic as particles are.

\footnotetext{
${ }^{10}$ For a nice explanation of this formula, see [6, p.376].
} 


\section{DISPOSITIONS IN NO-COLLAPSE INTERPRETATIONS}

In order to probe the consequences of the many-worlds interpretation for the dispositional or categorical nature of its postulated ontology, let us write the quantum state of the universe as a superposition of various "worlds": 11

$$
\left|\Psi_{\text {Universe }}>=\sum_{i} c_{i}\right| \Psi_{\text {World }-i}>
$$

with the usual condition $\sum_{i}\left|c_{i}\right|^{2}=1$.

In this view, each world behaves like a quasi-classical world of categorical properties, in which "indefinite" or fuzzy properties (i.e., superpositions) are absent. Consequently, there is a sense in which, in the many-world interpretation, quantum dispositions are "locally" reducible, or better, eliminable in each semi-classical world composing the big superposition.

However, since the whole universe is in a global state of superposition, we could as well conclude that, relative to the quantum state of the universe, which is a perfectly definite quantum state from a mathematical viewpoint, the physical properties of the universe are indefinite. Relative to the quantum state of the universe, we cannot claim that the moon has a definite position, given that the definiteness of such a property is only a relative matter, depending on, and varying with, different worlds, branches, minds, or perspectives, according to terminology. Consequently, in the manyworlds/many perspectives view it could be maintained that there is a sense in which the properties of the quantum universe (say, having a certain density at a certain time) can be understood as being irreducibly dispositional i.e., as being capable of having different definite "manifestations" in different worlds or branches or minds, all of them being equally real.

It could be objected that we should rather talk about the relational character of the properties in the quantum universe, since a dispositional property presupposes in principle the possibility of some sort of interaction for its manifestation, an interaction which in the many-world view seems absent. And this would be correct if no interacting correlation were envisaged.

However, that a dispositional reading of the many-worlds interpretations is not so implausible can be gathered from the notion of a centered-world developed by Simon Saunders [26]: if the world is centered on a human being, only perceived states are definite, and non-perceived ones are really superposed. Reality in itself is an entangled mess, and has the ungrounded, irreducible disposition to correlate to our brain states in such a way that we perceive the world as having definite properties. Never has an interpretation of a physical theory put more emphasis on the radical gap between the way the world is (reality) and how it appears to us: in this view, the above mentioned distinction between primary and secondary qualities evaporates, as all properties are secondary. ${ }^{12}$

Consequently, the question whether in the many-worlds view quantum dispositional properties are irreducible either (A) admits no definite answer or (B) it receives a

\footnotetext{
${ }^{11}$ Here I follow Lev Vaidman's notation [24].

${ }^{12}$ For the problem of the empirical coherence of Everett's type views of QM, see [27].
} 
positive one. Within the alternative (A), by focusing alternatively on the quantum state of the universe or on the single worlds composing it, we oscillate, respectively, from the irreducibility to the reducibility of quantum superpositions. In another, more plausible reading, however, which is closer to Everett's original intent of introducing many different perspectives on the same superposed indefinite universe, we have alternative (B): what is "really real" or mind-independent is the superposed state of the quantum universe, and the definiteness of the quasi-classical worlds is somehow less fundamental if not illusory, given that the classical world in which superpositions are eliminated is a world "centered" on the brains of human beings: the quantum state of the physical systems around us has the irreducible disposition to correlate in appropriate ways to human brains.

Also in this view, as it should be obvious, the definiteness of position in spacetime is a derived notion, since it is only in the single worlds that we can have definiteness of position and time of physical entities, something confirming our claim: the irreducibility of QM dispositions in a given interpretation is correlated to the fact that in that interpretation spacetime is a derived, non fundamental notion.

\section{Rovelli's Relational Version of QM}

According to Rovelli's relational view of QM [28], a quantum system $Q$ can be said to possess a certain property $q$ only relative to a system $S$; relative to another observing system (measuring apparatus) $S$ ', $Q$ and $S$ may be in an indefinite state, i.e., in a superposition. Such a view of QM is radically instrumentalist, insofar as it considers the $\Psi$-function as a mere bookkeeping devise, while focusing just on the sequence of actual measurement outcomes $q_{1}, q_{2}, \ldots, q_{\mathrm{n}}$. Such outcomes are to be regarded as the result of correlations of quantum systems with particular "observing physical systems" $S$, and no meaning is attached to the claim that an isolated quantum system $Q$ has intrinsic properties. In Rovelli's and Laudisa's words: «there is no meaning in saying that a certain quantum event has happened or that a variable of the system $S$ has taken the value $q$ : rather, there is meaning in saying that the event $q$ has happened or the variable has taken the value $q$ for $O$, or with respect to $O \ldots$ Quantum events only happen in interactions between systems, and the fact that a quantum event has happened is only true with respect to the systems involved in the interaction. The unique account of the state of the world of the classical theory is thus fractured into a multiplicity of accounts, one for each possible "observing” physical system.» [29, sect. 2]

Since in its context we cannot talk about intrinsic properties of physical systems, for our purpose it is important to stress that according to Rovelli's relational interpretation, the quantum world possesses no categorical properties at all. In this interpretation, the notion of "correlation" is so central that all properties of the quantum universe are not only relational, but also irreducibly dispositional, as they presuppose a physical interaction. In Rovelli's interpretation, it is meaningless to even think of categorical properties that ground the disposition to show certain values of position, spin etc.: while correlation events may be ascribed physical reality, the magnitudes that are the relata of the correlations cannot, as they are irreducibly dispositional. 
Also in the case of this interpretation, the presence of irreducible disposition is linked to the fact that spacetime emerges from, or is constituted out of, the innumerable correlation events between quantum systems and classical systems: there is no possibility of attaching categorical properties to microsystems prior to correlations. All properties are irreducibly dispositional.

\section{DISPOSITIONS IN BOHR'S INTERPRETATION OF QM}

Recently, historians have insisted that Bohr's view of QM should be differentiated from the so-called "Copenhagen interpretation", the latter being a sort of straw-man position created by philosophers [30]. By focusing just on Bohr's view, here I want by no means to suggest that Bohr's own interpretation is not afflicted by remarkable difficulties, in particular the lack of a precise distinction between the quantum and the classical domain.

Even if Redhead is probably right in thinking that according to Bohr it is simply meaningless to attribute a system whose state is not an eigenstate of the relevant observable any property before and independently of measurement [15, pp. 49-51], Bohr certainly believed in the reality or mind-independence of atomic systems (entity realism). In this sense, he was antirealist about QM as a theory, while being a realist about its theoretical entities: in a word, he was no instrumentalist. In addition, I think it is fair to claim that he defended a strong form of experimental dispositionalism, which is exactly what interests us here. The relationship between the principle of complementarity of non-commuting observables and the dispositional nature of quantum entities has been first stressed by Bohr, and has not been sufficiently noticed by physicists and philosophers writing on him.

According to Bohr, two properties (like being particle-like or being wave-like, or having a definite position $v s$. having a definite momentum) are complementary if and only if they are mutually exclusive and jointly exhaustive [31]. We say that they are mutually exclusive because, from the point of view of the classical language, after measurement they can be attributed to the same classical system at the same time only via a contradiction. In fact, complementary properties cannot be simultaneously revealed by the same experiment, given that any apparatus obeys classical physics. On the other hand, if we refer to a quantum system before measurement, the complementary properties must be regarded as jointly exhaustive, because any attempt at attributing a not-yet measured micro-system only one of the two properties would yield an incomplete description. For instance, an electron is neither a particle nor a wave, but has intrinsic, dispositional features to being both. The principle of complementarity is therefore at the root of the merely "potential" character of the quantum world before measurement.

In order to be coherent with his complementarity view of QM, in fact, Bohr claims that the quantum world must be irreducibly dispositional, where irreducible refers to his disbelief in hidden, deterministic variables grounding the indefinite state of the system before measurement, and dispositional refers to the holistic nature of the 
micro-system, the manifestation of properties being holistically depending on the kind of experiment one wants to perform.

Granting, as we should, that Bohr believed in the mind-independence of atoms and particles [32], the dispositional character of the quantum world is consequently an essential characteristic of his interpretation of QM. The main reason for this claim is given by the fact, often repeated by Bohr especially in his debate with Einstein, that the manifestation of the "properties" of quantum systems requires an experiment, and the nature of the experiment determines which "aspect" (which of the complementary properties) of the quantum system will be revealed.

Take the familiar apparatus for a two-slits experiment: do we want to observe the interference effects and thereby manifest the typical wave-like nature of the quantum system on the fluorescent screen behind the slits? Then, as instrumentalist followers of Bohr usually put it, we must renounce to have any information about its particle-like aspect, for instance by trying to find out which slit the particle went through. I would suggest that in order to remain faithful to Bohr's entity realism, we should put the matter more realistically: by deciding to perform an experiment on the wavelike aspect, we must somehow destroy a dispositional aspect of the system, related to its particle-like, complementary disposition.

For our purposes, it is important to note that the quantum system's wavelike manifested property causally depends on its interaction with the apparatus, and therefore on what we decided to measure. According to Bohr's interpretation, we cannot assume that there is a categorical basis for the manifestation of the wavelike property before a measurement interaction, since this would be equivalent to assume that the wavelike aspect was there all along before measurement, at the exclusion of the particle-like, complementary aspect. But an unmeasured system has both dispositions. If we assumed such a categorical basis for the wave-like aspect of the system, we could not explain why, if we closed one of the two slits, the interference effects due to the superposition would be lost, and on the screen we would observe just an enlarged image of the slit.

Using the stipulations above, we are now in the position to understand Bohr's refusal of hidden variable theories (and his consequent belief in the completeness of $\mathrm{QM}$ ) as being equivalent to the claim that there is no categorical basis for neither of the dual, "complementary" aspects of the quantum systems. Furthermore, to the extent that QM is, as Bohr thought, complete, there is no possessed (non dispositional) property before measurement (when the system is not in an eigenstate of the observable), since it is meaningless to attribute any property to a quantum system independently of a measurement context.

In a word, Bohr's entity realism gives us a belief in irreducible dispositions, a language that Bohr himself never used explicitly, but to which, I submit, he would not have objected at all: given his belief about the existence of theoretical entities, he had to take a stance about their way of existence vis à vis their dynamical features, and irreducible dispositionality would serve his purpose well. Quantum properties, according to a minimally realistic reading of Bohr, are irreducibly dispositional because experiments create, at least in part the dynamical properties they measure. ${ }^{13}$

\footnotetext{
${ }^{13}$ Mass, spin and charge are intrinsically possessed also according to Bohr.
} 


\section{CONCLUDING REMARKS}

It is time to draw our take-home lesson from the quick and necessarily brief overview of some of the main interpretations of the formalism of QM.

(1) If interpreting means trying to figure out what is out there in the physical world, dispositions are ubiquitous in all such interpretations, and yield a sort of unifying language for views that are widely different among them, as Bohr's and Bohm's are;

(2) With the important exception of Bohm's minimalist interpretation sketched above, all such dispositions are irreducible to categorical, non-contextually possessed properties;

(3) the reason why the non-Bohmian views are committed to irreducible dispositions is importantly different in GRW on the one hand, and in the non-collapse views and Bohr on the other.

(4) In the former case (GRW), we have irreducibly stochastic processes that have no categorical basis because such processes are fundamentally uncaused. In the latter case, we have irreducible dispositions because the quantum world before measurement (when the state is not an eigenstate of the relevant observable) is regarded as devoid of any definite, intrinsic property whatsoever. What is emphasized in the former view is a new physical process, regarded as responsible for the solid, perceived definiteness of the classical world. In the latter views, metaphysical considerations about irreducible dispositionality are upheld to try to prevent any further physical inquiry into the nature of measurement interactions, or any deeper explanations of the reason why Born's $|\Psi|^{2}$ holds and works. If categorical bases of dispositions are regarded as lacking, no such physical progress is going to be possible in principle. The possibility that an ideological, obscurantist role of dispositionality be hidden in the latter views should not be excluded at all;

(5) a common element between the views defending irreducible dispositions could be linked to their disavowal of the possibility of a spatiotemporal description of a quantum system prior to a collapse, for collapse views, prior to a correlation with any other system, for the many-world theorists and Rovelli, or prior to a measuring process realized via a classical apparatus for Bohr. The role of an event ontology in the various interpretation of QM should be studied more accurately than it has been done so far [33]

(6) Despite one's inclinations toward realism about dispositions, it is one thing to claim that dispositions, as in the classical case, are identical with, reducible to, or explainable by other categorical properties, or even other more microscopic dispositions. It is quite another thing to have to swallow irreducible dispositions as in Bohr's or the Everettian views. It must be granted that introducing irreducible physical dispositions is implicitly admitting that there is something we don't understand. Admitting an in-principle lack of any categorical basis to which dispositions could be reduced, in both the non-collapse views and Bohr's seems a way to surrender to mystery. 


\section{ACKNOWLEDGMENTS}

I thank Federico Laudisa and Nino Zanghì for several discussions and for the generosity with which they offered advice.

\section{REFERENCES}

1. S. Kochen S. and E. Specker, Journ. of Math. and Mech., 18, 1015-1021 (1967).

2. G.C. Ghirardi and R. Grassi, Stud.Hist.Phil.Sci. 25, 397-423 (1994).

3. D. Dürr, S. Goldstein and N. Zanghì, "Bohmian Mechanics as the Foundations of Quantum Mechanics", in Bohmian Mechanics and Quantum Theory: an Appraisal, edited by J. Cushing, A. Fine and S. Goldstein, Dordrecht: Kluwer, 1996, pp. 21-44.

4. J. von Neumann, Foundations of Quantum Mechanics, Princeton NJ.: Princeton University Press, 1955.

5. E. Wigner, "Remarks on the Mind-Body Question", in Symmetries and Reflections, Bloomington IN: Indiana University Press, 1967, pp. 171-184.

6. N. Zanghì, "Un viaggio nel mondo quantistico“, in V. Allori, M. Dorato, F. Laudisa, N. Zanghì, La natura delle cose, Roma: Carocci, 2005, pp. 229-394.

7. M. Lange, An Introduction to the Philosophy of Physics, Oxford: Blackwell, 2002.

8. K. R. Popper, Quantum Theory and the Schism in Physics, edited by W. W. Bartley, London: Hutchinson, 1982.

9. M. Daumer, D. Dürr, S. Goldstein, and N. Zanghì, Erkenntnis 45, 379-397 (1996).

10. R. Clifton R. and C. Pagonis, Found. Phys., 25, 2, 281-296 (1995).

11. D. Bohm and B. J. Hiley, The Undivided Universe: An Ontological Interpretation of Quantum Theory, London: Routledge \& Kegan Paul, 1993.

12. W. Heisenberg, Physics and Philosophy: the Revolution in Modern Science, New York: Harper and Row, 1958.

13. H. Margenau, Physics Today, 7, (1954), pp. 6-13.

14. N. Maxwell, Brit. Journ. Philos. Scie., 39, pp. 1-50 (1988).

15. M. Redhead, Incompleteness, Non-locality and Realism, Oxford: Clarendon Press, (1987).

16. M. Suárez, Erkenntnis 61, 1-16 (2004).

17. M. Suárez, Brit. Journ. Phil. Sci. 55 (2), 219-255 (2004).

18. N. Grossman, Phil. of Sci. 39, 451-460, (1972).

19. P. Milne, Brit. Journ. Phil. Sci. 36 (2), 66-70, (1985).

20. A. Bassi and G. C. Ghirardi, Physics Report, 379, 257-426, (2003).

21. G.C. Ghirardi, A. Rimini A. and T. Weber, Phys. Rev. D 34, 470-491 (1986).

22. J. Bell, “Are There Quantum Jumps?,” in Speakable and Unspeakable in Quantum Mechanics, Cambridge: Cambridge University Press, 1987, pp. 201-212.

23. M. Friedman, The Journal of Philosophy, 71, 5-19, (1974).

24. P. Kitcher, The Journal of Philosophy 73, 207-212, (1976).

25. L. Vaidman, “The Many-Worlds Interpretation of Quantum Mechanics”, in Stanford Encyclopedia of Philosophy, (2002), http://plato.stanford.edu/entries/qm-manyworlds/

26. S. Saunders, Synthese, 102, 235-266, (1996).

27. J. Barrett, The Quantum Mechanics of Minds and Worlds, Oxford: Oxford University Press, 1999.

28. C. Rovelli, Intern. Journ. Theor. Phys. 35, 1637-1678, (1996).

29. F. Laudisa and C. Rovelli, "Relational Quantum Mechanics", in Stanford Encyclopedia of Philosophy, http://plato.stanford.edu/entries/qm-relational/

30. D. Howard, Phil. Sci, 71: 669-682 (2004).

31. D. Murdoch, Niels Bohr's Philosophy of Physics, Cambridge : Cambridge University Press, 1987.

32. J. Faye, Niels Bohr: His Heritage and Legacy, Dordrecht: Kluwer, 1991.

33. M. Dorato, "Becoming, Events and the Ontology of Quantum Physics", in R. Buccheri, A. Elitzur and M. Saniga (eds.), Endophysics, Time, Quantum and the Subjective, Singapore: World Scientific, 2005, pp .273289. 